\title{
SOME BIOCHEMICAL IMPLICATIONS IN AUTISM SPECTRUM DISORDER
}

\author{
Cristiane Pinheiro Lázaro*, Milena Pereira Pondé**, Luiz Erlon A. Rodrigues*** \\ Corresponding author: Luiz Erlon A. Rodrigues - erlon@svn.com.br \\ * Doctoral student in Medicine and Human Health, Bahiana School of Medicine and Public Health, Salvador, Bahia, Brazil \\ ** PhD, Adjunct Professor, Interdisciplinary Research Laboratory in Autism, Bahiana School of Medicine and Public Health, \\ Salvador, Bahia, Brazil \\ ***PhD, Professor of Medical Biochemistry, Basic Research Laboratory, Bahiana School of Medicine and Public Health, \\ Salvador, Bahia, Brazil
}

\begin{abstract}
Autism spectrum disorder (ASD) affects 0.6 to $1 \%$ of the population worldwide. It is characterized by a deficit in communication and social interaction, and is associated with restricted and repetitive behavior patterns. Stereotypes include inflexible adhesion to specific non-functional routines and rituals and a persistent concern with parts of objects rather than the object as a whole. Up to the present time, there are no specific tests that permit a laboratory diagnosis of the disorder to be carried out, and the syndrome is confirmed by clinical observation in the first 36 months of the patient's life. Clinical manifestations such as epilepsy, mental retardation, sleep disorders, hyperactivity, irritability and auto- and heteroaggressiveness may alter the patient's prognosis. Around $50 \%$ of children with ASD fulfill the criteria for attention deficit hyperactivity disorder (ADHD). Symptoms of oppositional defiant disorder (ODD) associated with autism appear to indicate a distinct phenotype requiring specific therapeutic measures. ASD is not a discrete nosological entity but, rather, a multifactorial syndrome associated with different phenotypic and biological presentations. Various disorders such as pathologies of the gastrointestinal tract have been linked to ASD, not only insofar as causality is concerned but also with respect to their role in aggravating the disease. Other associated disorders include lesions in physiological processes such as the redox metabolism, mitochondrial dysfunction and enzymatic regulation of essential metabolites. Currently, studies on direct and indirect markers of mitochondrial metabolism associated with anomalies found in the brain of these patients point to the possibility of these markers being used as tools with which to reach a diagnosis that would be laboratory-based rather than merely clinical. Keywords: Autism Spectrum Disorder; Oxidative Stress; Mitochondrial Dysfunction.
\end{abstract}




\section{INTRODUCTION}

Autism spectrum disorder (ASD) affects 0.6 to $1 \%$ of the population worldwide. Its onset occurs at an early age and the symptoms and associated functional impairment persist throughout life. ${ }^{(1)}$ The typical symptoms begin to be noted in the first three years of life and affect the child's development. Up to the present time, there are no specific tests that would allow the diagnosis to be laboratory-based. Diagnosis of the syndrome is confirmed by clinical observation during the first 36 months of the child's life ${ }^{(2)}$ In more severe cases, the symptomatology manifests itself before the child completes his/ her first year of life, although there are cases in which, according to the parents, no manifestations whatsoever were present in the apparently normal first 24 months of life. ${ }^{(3)}$

According to the Diagnostic and Statistical Manual of Mental Disorders, fifth edition (DSM5), ASD is characterized as persistent deficits in social communication and social interaction across multiple contexts, associated with restricted patterns of behavior, interests or activities. Difficulties in spontaneity, imitation and social games are common findings, as are deficits in social-emotional reciprocity. ${ }^{(4,5)}$ In relation to communication, the development of speech is delayed and language use is stereotyped and repetitive, with no ability to initiate or sustain a conversation. ${ }^{(6)}$ In addition, in cases in which communication is affected to a lesser extent, pronoun reversal and immediate and delayed echolalia may be present. ${ }^{(5,6)}$ Another area affected refers to restrict, repetitive patterns of behavior, interests and activities. These may be manifest as inflexible adherence to specific, nonfunctional routines and rituals and in persistent concern with parts of objects rather than the object as a whole. ${ }^{(3,4)}$ When simple changes to routine activities are proposed, manifestations of resistance and distress are common. ${ }^{(2)}$

Some clinical manifestations such as epilepsy, mental retardation, sleep disorders, hyperactivity, irritability, auto- and hetero-aggressiveness may modify the prognosis of the affected child. (7) ASD is often associated with mental retardation, epilepsy and genetic complications, particularly the Fragile $X$ syndrome. ${ }^{\left({ }^{(8)}\right.}$ According to Pondé et al., (9) around $50 \%$ of children with a diagnosis of autism fulfill the criteria for a diagnosis of attention deficit hyperactivity disorder (ADHD). Little information is available on the learning difficulties associated with ADHD symptoms in autistic children; however, recent studies suggest a strong association between symptoms of ADHD and learning difficulties. ${ }^{(10,11)}$ These studies make it reasonable to speculate that the presence of ADHD symptoms in patients with ASD may hamper learning. Nevertheless, treatment of ADHD symptoms with methylphenidate (Ritalin), a drug much used at the moment for the treatment of ADHD, has been found to be less effective and the rate of side effects is higher. ${ }^{(12)}$ This inadequacy of the treatment demands a more specific investigation.

Other symptoms associated with ASD such as aggressiveness, oppositional behavior and alterations in behavior may also affect the possibility of the child accessing socio-educational measures and, consequently, his/her social adaptation and prognosis. According to Gadow et al.,, ${ }^{(13)}$ symptoms of oppositional defiant disorder (ODD) associated with autism appear to indicate a different phenotype, requiring specific treatment. Auto- and heteroaggressive behavior in individuals with ASD appears to be associated with a cognitive deficit and a poorer prognosis insofar as social adaptation is concerned. (14) Furthermore, irritability and the aforementioned types of aggressiveness may result in physical risks to the child and most often interfere negatively in his/her regular contacts with the rest of society, in his/her access to education and, in addition, require pharmacological interventions to contain disruptive behavior ${ }^{(15,16)}$

Since ASD is a multifactorial syndrome, its origin is as yet unknown. Autism is not a discrete nosological entity but, rather, a broad syndrome that varies both with respect to the intensity of central symptoms and to the presence of associated symptoms, thus generating a huge variety of clinical presentations. Therefore, by taking causal factors associated with ASD into account, the different 
phenotypic and neurobiological presentations must be considered.

According to Samsam et al., ${ }^{(17)}$ many biological disorders are associated with ASD, not only insofar as causality is concerned but also with respect to the severity of the disease. Of these disorders, those authors emphasized pathologies of the gastrointestinal tract, including dysbiosis, inflammatory bowel disease, exocrine pancreatic insufficiency, coeliac disease, poor digestion, poor absorption, food intolerance and food allergies, all of which can result in malnutrition. On the other hand, ASD is strongly associated with deficiencies in basic physiological processes, the most important of which include the mitochondrial redox metabolism and abnormalities in the regulation of essential metabolites such as folic acid, tetrahydrobiopterin, glutathione, cholesterol, carnitine and branchedchain amino acids. ${ }^{(18)}$ Part of these studies was conducted using peripheral markers of metabolism; however, various anomalies were also found in the brains of these individuals, principally those associated with mitochondrial dysfunction and oxidative stress. ${ }^{(19)}$

\section{OXIDATIVE STRESS}

Mitochondria are cytoplasmic organelles surrounded by a double membrane, and their principal function is to synthesize adenosine triphosphate (ATP), the most important energy transporter in all aerobic cells. Its principal substrate, acetylcoenzyme $A$ (Acetyl-CoA), is generated from glucose metabolism, from the D-oxidation of fatty acids and, partially, from glycogenic amino acids, which are metabolized by the Krebs cycle and by the oxidative metabolism of fatty acids. ${ }^{(20)}$ The Krebs cycle generates nicotinamide-adenine dinucleotide $(\mathrm{NADH})$ and flavin-adenine dinucleotide (FADH2), both reduced, that transport hydrogen to the mitochondrial respiratory chain where a series of oxidation-reduction reactions occur, leading to the synthesis of ATP by the oxidative phosphorylation of ADP. ${ }^{(20)}$
The biochemical signature of a mitochondrial lesion appears in the form of metabolites that divert hydrogen or electrons from the respiratory chain. Since no cell survives without an effective defense, the mitochondria represent the organism's frontline of defence, since they are directly involved in activating pathways linked to innate immunity and to the control of inflammatory responses. ${ }^{(21)}$ Therefore, one of the most important functions of the mitochondria refers to the metabolic response to lesions. ${ }^{(22)}$

Since they are the only organelles in the cells of mammals with their own genome, they possess an entire electron transport chain codified by mitochondrial DNA (DNAmt) and by nuclear DNA (DNAn), which also codify the mitochondrial enzymes that participate in the oxidation of carbohydrates and fatty acids. Therefore, any mutation that occurs in the genome may hamper mitochondrial function and lead to a variety of deficiencies. ${ }^{(23)}$ Furthermore, the electron transport chain constitutes the principal source of reactive oxygen species (ROS), (24) which are normally detoxicated by the enzymes superoxide dismutase, glutathione oxidoreductase (GOR) and catalase. For the production of GOR, mitochondria depend on cytoplasmic metabolism. When the level of GOR decreases, the cells consequently become more vulnerable to oxidative stress. ${ }^{(18)}$ Nevertheless, when there is an increase in ROS, apoptosis begins in two ways: through extrinsic pathways (cytoplasmic) or intrinsic pathways (mitochondrial). ${ }^{(25)}$ This process permits activation of another mechanism of mitochondrial defense that removes cells that are no longer necessary and the lesions caused by pathogenic agents. ${ }^{(26)}$

Mitochondria contain a proteome with 1500 proteins, and around 1000 of these proteins exert catalytic functions in the cell metabolism, acting as enzymes. Under normal physiological conditions, nutrients and metabolic substrates are regulated by several of these enzymes. ${ }^{(24)}$ However, when their concentrations change as the result of infections or other aggressions, the mitochondria detect the incompatibility between the concentration and demand in the affected tissue, divert the flow of 
electrons and reduce oxygen consumption. ${ }^{(18)}$ When this consumption falls, oxygen concentration in the cell increases, the reduction-oxidation processes are changed and the biosynthesis of polymers (DNA, RNA, complex lipids, proteins and polysaccharides) is interrupted due to the reduction in the NADPH/ NADP+ ratio and in the free energy available. ${ }^{(26)}$

Under greater oxidizing conditions, the uptake of electrons increases as a function of the growing presence of molecular oxygen. From this, there is oxidation of the iron-sulphur clusters and of the redox-sensitive sites in numerous proteins that protect the cell membrane from new attacks. ${ }^{(24)}$ The effect of oxidative stress on mitochondrial function in individuals with autism spectrum disorder suggests that there is an imbalance between ROS generation and the antioxidant defense mechanism in the organism of these individuals. This would be motivated by various enzymatic alterations such as: reduction of glutathione peroxidase (GPx) in plasma ${ }^{(27)}$ and in the erythrocytes; ${ }^{(27,28)}$ a smaller proportion of reduced glutathione in relation to oxidized glutathione (GSSG) in the cytoplasm; ${ }^{(24)}$ a reduction in catalase activities ${ }^{(18)}$ and in superoxide dismutase (SOD) in the erythrocytes. ${ }^{(28)}$

Superoxide dismutase, catalase and glutathione peroxidase, considered primary enzymes, are directly involved in eliminating ROS and depend on glutathione reductase, glucose-6-phosphate dehydrogenase and 6-phosphogluconate dehydrogenase. As secondary antioxidants, they help maintain reduced glutathione and NADPH levels constant. ${ }^{(20)}$ In addition, to assure that this entire mechanism functions well, these enzymes need metal cofactors (micronutrients) such as selenium, iron, copper, zinc and manganese. ${ }^{(20)}$

Nevertheless, the aforementioned defense mechanism may be compromised in autistic individuals. The dietary intake of these individuals is replete with restrictions due to their oral, olfactory and tactile hypersensitivity, ${ }^{(29)}$ which is related to texture, color, taste, the shape of food, difficulties in chewing and swallowing, ${ }^{\left({ }^{\circ}\right)}$ and gastrointestinal disorders. ${ }^{(31)}$ Some studies show that nutritional deficiencies $^{(32)}$ and even malnutrition are a consequence of this condition. ${ }^{33)}$
On the other hand, neurones are extremely susceptible to oxidative stress and various factors limit their capacity to support oxidation. ${ }^{(34)}$ These factors include a) a high level of substrates that are easily oxidized (polyunsaturated fatty acids); b) relatively low levels of antioxidants (GOR and antioxidant enzymes); and c) the endogenous generation of ROS through reactions that lead to the endogenous production of nitric oxide (NO). ${ }^{(34)}$ Furthermore, the nervous system is vulnerable to oxidative damage due to the fact that replication of its cells is difficult, since, once damaged, they become permanently dysfunctional, in addition to interfering with the mechanism of apoptosis. ${ }^{25)}$ Rose et al. ${ }^{(35)}$ evaluated frozen samples of the cerebellum and temporal cortex of autistic individuals and found a reduction in the level of GOR and in the GOR/GSSG ratio, and a reduction in the activity of mitochondrial aconitase (a biomarker of superoxide production), in addition to an increase in oxidative damage in the mitochondrial DNA protein. These results show a lower antioxidant capacity and an increase in oxidative stress in the brain of these individuals, which may have functional consequences in terms of a chronic inflammatory response and in increasing the production of mitochondrial superoxide and oxidative damage to DNA proteins.

\section{MITOCHONDRIAL DYSFUNCTION}

Various diseases are associated with mutations in mitochondrial DNA (DNAmt) and nuclear DNA (DNAn) result in functional defects in these organelles or in their inability to minimize the consequences of oxidative stress. ${ }^{(36)}$ DNA mutations and the consequent degradation of proteins, lipids and nucleic acids, most probably caused by the action of ROS, are probably involved in the genesis of mitochondrial diseases. Nevertheless, in accordance with the oxidative shielding mechanism, ${ }^{(24)}$ the functional and metabolic defects occur prior to the increase in ROS and prior to the oxidative changes. The increase in the production of these radicals may occur in response to the disease rather than seeming to be the cause. Therefore, it is 
not the right oxidative changes to which treatment should be directed but, rather, to the metabolic conditions that create them. On the other hand, spacial proximity of DNAmt to these radicals produced in the electron transport chain makes it singularly susceptible to mutations, particularly when the electron transport chain is dysfunctional. ${ }^{(36)}$

Cells with a more active metabolism, which physiologically need more energy, such as the muscles, the digestive tract and the neurones, depend to a greater extent on mitochondrial metabolism. Neuronal synapses that biosynthesize the neurotransmitters require more energy; therefore, they are more sensitive to mitochondrial dysfunction (MD) ${ }^{(34)}$ and their phenotypic presentation is broader. Their diagnosis is based on various clinical, histological and biochemical parameters, on neuroimaging and some enzymatic findings.

Up to the present time, there is no specific biomarker with which to identify mitochondrial dysfunction. Nevertheless, some laboratory measurements have been described as direct biomarkers (lactate dehydrogenase, pyruvate decarboxylase, the lactate/pyruvate ratio, ubiquinone, alanine, the alanine/lysine ratio and acylcarnitine) and indirect biomarkers (creatine phosphotransferase [CPK], carnitine, aspartate aminotransferase [AST] and plasma ammonia). $(18,37,38)$ These markers may be abnormal for various reasons: mitochondrial dysfunction hampers aerobic respiration, leading to a reduction in the function of the Krebs cycle, which results in an increase in pyruvate, in NAD+ and in other intermediate metabolites. Pyruvate, which is reduced to lactate and transamination to alanine, promotes an increase in these metabolites in plasma. ${ }^{(18)}$ Inhibition of aerobic respiration also hampers the $\beta$-oxidation of fatty acids and consequently increases acylcarnitine levels. Cytoplasmic glycolysis increases when aerobic respiration is not enough to meet the demands of cellular energy. Since lactate is one of the final products of this glycolysis, the inhibition of aerobic respiration increases lactate even further. These results suggest that evaluating lactacidemia could be useful for reaching a preliminary diagnosis of mitochondrial dysfunction. ${ }^{(25)}$

Indirect markers may also be abnormal. Depletion of total and free carnitine may occur as a consequence of the failure to convert fatty acids. (37) In conditions of deficient oxygenation, ammonia is produced when adenosine monophosphate is transformed into inosine monophosphate by the enzyme adenosine deaminase (ADA) in order to increase the biosynthesis of ATP. Since the biosynthesis of urea is partially located in the mitochondria, mitochondrial dysfunction may result in secondary dysfunction of ureogenesis and an increase in ammonia. Furthermore, the integrity of tissues that require a lot of energy, such as the muscles, may be compromised following mitochondrial dysfunction, resulting in increases in the indicators of tissue damage such as creatine phosphotransferase, lactate dehydrogenase, AST and ALT. ${ }^{(38)}$

Mitochondrial dysfunction can be considered to be primary or secondary. Primary mitochondrial dysfunction generally refers to dysfunction caused by a genetic defect directly involved in the function of mitochondrial systems responsible for ATP production, while secondary mitochondrial dysfunction refers to other abnormalities in the metabolic or genetic processes. ${ }^{(36)}$ Secondary causes include certain drugs; carboxylic acids such as propionic acid; high concentrations of tumor necrosis factor alpha (TNF $)$; cerebral folate deficiency; malnutrition; vitamin $\mathrm{B} 6$ and iron deficiency; increased nitric oxide; deficiency of reduced glutathione; oxidative stress; and exposure to environmental toxins. ${ }^{(26)}$ In some individuals, results are consistent with mitochondrial dysfunction; however, they do not have any identifiable genetic defect or do not fulfill the criteria for a probable diagnosis. It is possible that these individuals have secondary mitochondrial dysfunction or an as yet unidentified genetic abnormality.

Mitochondrial dysfunction has been associated with psychiatric ${ }^{(23)}$ and neurological(39) diseases and with autism spectrum disorder. ${ }^{(34)}$ In the meta-analysis conducted by Rossignol and Frye, ${ }^{(18)}$ 
a prevalence of $5 \%$ of mitochondrial dysfunction was found in children with ASD with abnormal values of direct biomarkers (lactate, pyruvate and the lactate/pyruvate ratio) and indirect biomarkers (carnitine, ammonia, creatine phosphotransferase and AST). In that same review, children with ASD and mitochondrial dysfunction had several clinical characteristics such as regression in development, convulsions, delayed motor development, gastrointestinal alterations and a greater prevalence of increased lactate and pyruvate compared to the population with autism alone.

Finally, other biochemical abnormalities can contribute to secondary mitochondrial dysfunction. Several studies $(35,37,38,40)$ have reported lower mean glutathione levels in children with ASD compared to controls. From the point of view of mitochondrial function, this finding is very concerning, since that substance combats the adverse effects of tumor necrosis factor alpha (TNF $\alpha$ ), a proinflammatory cytokine that inhibits mitochondrial function. Corroborating this finding, the study conducted by Rose et al. ${ }^{(41)}$ reported higher TNF $\alpha$ levels in lymphocytes, in cerebrospinal fluid and in the brains of autistic individuals compared to a control group.

\section{CONCLUSION}

In view of the above and of the important role of biochemical factors in establishing ASD, further studies should be conducted with the objective of identifying more specific markers that can help diagnose this disorder and monitor therapy that is still in development for children with ASD.

\section{REFERENCES}

1. Simonoff E, Pickles A, Charman T, Chandler S, Loucas T, Baird G. Psychiatric Disorders in Children With Autism Spectrum Disorders: Prevalence, Comorbidity, and Associated Factors in a Population-Derived Sample. J Am Acad Child Adolesc Psychiatry. 2008; 47(8):921-9.
2. Ministério da Saúde. Secretaria de Atenção à Saúde. Departamento de Ações Programáticas Estratégicas. Diretrizes de Atenção à Reabilitação da Pessoa com Transtornos do Espectro do Autismo - Brasília: Ministério da Saúde; 2013. p.74 (Série F. Comunicação e Educação em Saúde).

3. Sacrey LA, Germani T, Bryson SE, Zwaigenbaum L. Reaching and grasping in autism spectrum disorder: a review of recent literature. Front Neurol.2O14;23(5):1-12.

4. Pelphrey KA, Yang DY, McPartland JC. Building a social neuroscience of autism spectrum disorder. Curr Top Behav Neurosci. 2014;16:21533.

5. Bird G, Cook R. Mixed emotions: the contribution of alexithymia to the emotional symptoms of autism. Transl Psychiatry.2O13;3(7):e285.

6. Foti F, De Crescenzo F, Vivanti G, Menghini D, Vicari S. Implicit learning in individuals with autism spectrum disorders: a meta-analysis. Psychol Med.2014;15:1-14.

7. Novaes MC, Pondé MP, Freire ACC. Control of psychomotor agitation and agressive behavior in patients with autistic disorder: a retrospective chart review. Arq Neuropsiquiatr. 2008;66(3B):646-651.

8. Yu TW, Berry-Kravis E. Autism and fragile $x$ syndrome. Semin Neurol. 2014;34(3):258-65.

9. Pondé MP, Novaes CM, Losapio MF. Frequency of symptoms of attention deficit and hyperactivity disorder in autistic children. Arq Neuropsiquiatr. 2010;68(1):103-106.

10. Pondé MP, Cruz-Freire AC, Silveira AA. Relationship between learning problems and attention deficit in childhood. J Atten Disord. 2012;16(6):505-9.

11. Talero-Gutierrez C, Van Meerbeke AV, Reyes RG. A Clinical Study of ADHD Symptoms With Relation to Symptoms of Learning Disorders in Schoolchildren in Bogota, Colombia. J Att Disord. 2012;16(2):157-163.

12. RUPP (Research Units on Pediatric Psychopharmacology Autism Network). Randomized, controlled, crossover trial of methylphenidate in pervasive developmental 
disorders with hyperactivity. Arch Gen Psychiatry.2005;62(11):1266-74.

13. Gadow KD, DeVincent CJ, Drabick DA. Oppositional defiant disorder as a clinical phenotype in children with autism spectrum disorder. J Autism Dev Disord.2008;38:1302-1310.

14. Minshawi N. Behavioral Assessment and treatment of self-Injurious behaviour in autism. Child Adolesc Psychiatr Clin N Am. 2008;17:875-886.

15. Moura AMR, Menezes APA, Pondé MP. Atualização da farmacoterapia do autismo. In: Garcia R, Pondé MP, Lima MG. Atualização em Psicoses. São Paulo: Phoenix; 2004. p. 235-244.

16. Stingle KA, McDougle CJ. Pharmacotherapy of irritability in pervasive developmental disorders. Child Adolesc Psychiatr Clin N Am. 2008;17:729-752.

17. Samsam M, Ahangari R, Naser SA. Pathophysiology of autism spectrum disorders: Revisiting gastrointestinalinvolvement and immune imbalance. World J Gastroenterol. 2014;2O(29):9942-9951.

18. Rossignol DA, Frye RE. Mitochondrial dysfunction in autism spectrum disorders: a systematic review and meta-analysis. Mol Psychiatry.2012;17,290-314.

19. Cubała-Kucharska M. The review of most frequently occurring medical disorders related to aetiology of autism and the methods of treatment. Acta Neurobiol Exp.2010;70:141-146.

2O. Kamoun P, Lavoinne A, de Verneuil H. Bioquímica e biologia molecular. Tradução João Paulo e Campos e Paulo A. Motta. Rio de Janeiro: Guanabara Koogan; 2006.

21. Naviaux RK, Zolkipli Z, Wang L, Nakayama T, Naviaux JC, Le TP, Schuchbauer MA et al. Antipurinergic therapy corrects the autism-like features in the poly (IC) mouse model. PLoS One.2013;8:e5738.

22. West AP, Shadel GS, Ghosh S. Mitochondria in innate immune responses. Nat Rev Immunol. 2011;1:389-4O2.

23. Streck EL, Gonçalves CL, Furlanetto CB, Scaini G, Dal-Pizzol F, Quevedo J. Mitochondria and the central nervous system: searching for a pathophysiological basis of psychiatric disorders. Rev. bras. psiquiatr. 2014;36(4):156167.

24. Naviaux RK. Oxidative shielding or oxidative stress? J Pharmacol Exp Ther. 2O12; 342:608618.

25. Karbowski M. Mitochondria on guard: role of mitochondrial fusion and fissionin the regulation of apoptosis. Adv Exp Med Biol. 2010;687:131142.

26. Davis RE, Williams M. Mitochondrial Function and Dysfunction: An Update. J Pharmacolol Exp Ther. 2012;342(3):598-607.

27. Frustaci A, Neri M, Cesario A, Adams JB, Domenici E, Dalla Bernardina B, Bonassi S. Oxidative stress-related biomarkers in autism: systematic review and meta-analyses. Free Radic Biol Med. 2O12;15(10):2128-41.

28. El-Ansary AK, Ben Bacha A, Kotb M. Etiology of autistic features: the persisting neurotoxic effects of propionic acid. J Neuroinflammation.2012;24;9:74.

29. Cermak SA, Curtin C, Bandini LG. Food selectivity and sensory sensitivity in children with autism spectrum disorders. J Am Diet Assoc. 2010;110(2):238-246.

30. Diolordi L, Del Balzo V, Bernabei P, Vitiello V, Donini LM. Eating habits and dietary patterns in children with autism. Eat Weight Disord. 2014;19(3):295-301.

31. Chaidez V, Hansen RL, Hertz-Picciotto I. Gastrointestinal problems in children with autism, developmental delays or typical development. J Autism Dev Disord. 2014;44(5):1117-27.

32. Hyman SL, Stewart PA, Schmidt B, Cain U, Lemcke N, Foley JT, Peck R et al. Nutrient intake from food in children with autism. Pediatrics.2O13;13O(2):S145-53.

33. Kawicka A, Regulska-llow B. How nutritional status, diet and dietary supplements can affect autism. A review. Rocz Panstw Zakl Hig.2013;64(1):1-12.

34. Rossignol DA, Frye RE. Evidence linking oxidative stress, mitochondrial dysfunction, and inflammation in the brain of individuals with autism. Front Physiol. 2014;22(5):150. 
35. Rose S, Melnyk S, Pavliv O, Bai S, Nick TG, Frye RE, James SJ. Evidence of oxidative damage and inflammation associated with low glutathione redox status in the autism brain. Transl Psychiatry. 2012;1O(2):el34.

36. Davis RL, Sue CM. The genetics of mitochondrial disease. Semin Neurol. 2011;31(5):519-30.

37. Frye RE. 15q11.2-13 duplication, mitochondrial dysfunction, and developmental disorders. J Child Neurol. 2009;24:1316-1320.

38. Haas RH, Parikh S, Falk MJ, Saneto RP, Wolf $\mathrm{NI}$, Darin $\mathrm{N}$ et al. The in-depth evaluation of suspected mitochondrial disease. Mol Genet Metab. 2008;94:16-37.
39. Duarte JM, Schuck PF, Wenk GL, Ferreira GC. Metabolic disturbances in diseases with neurological involvement. Aging Dis.2013;30;5(4):238-55.

4O. Palmieri L, Persico AM. Mitochondrial dysfunction in autism spectrum disorders: cause or effect? Biochim Biophys Acta 2010;1797(67):1130-7.

41. Rose S, Frye RE, Slattery J, Wynne R, Tippett M, Pavliv O, Melnyk S et al. Oxidative stress induces mitochondrial dysfunction in a subset of autism lymphoblastoid cell lines in a well-matched case control cohort. PLoS One.2014;8;9(1):e85436. 